\title{
Triviality of fibered Calabi-Yau manifolds without singular fibers
}

\author{
VAlentino Tosatti And Yuguang Zhang
}

\begin{abstract}
In this paper, we show that if a compact Kähler manifold with trivial canonical bundle is the total space of a holomorphic fibration without singular fibers, then the fibration is a holomorphic fiber bundle. In the algebraic case, the fibration becomes trivial after a finite base change.
\end{abstract}

\section{Introduction}

Let $X$ be a compact Kähler manifold with $c_{1}(X)=0$ in $H^{2}(X, \mathbb{R})$, which we will call a Calabi-Yau manifold. Thanks to a fundamental theorem of Yau [42], the class of Calabi-Yau manifolds is precisely the class of compact Kähler manifolds which admit Ricci-flat Kähler metrics. Furthermore, $X$ Calabi-Yau implies that the canonical bundle $K_{X}$ is holomorphically torsion [4]. Assume that there is a holomorphic submersion with connected fibers $f: X \rightarrow Y$ to a compact Kähler manifold $Y$. Let $n=\operatorname{dim} X, m=\operatorname{dim} Y$ and assume that $0<m<n$. Then every fiber $X_{y}=f^{-1}(y)$ is a Calabi-Yau manifold of dimension $n-m$. In other words, $X$ is Calabi-Yau and is also the total space of a family of Calabi-Yau manifolds over $Y$.

In the case when $f$ is allowed to have singular fibers, this is the setup of the first author's paper [39], where it is proved that certain families of Ricci-flat Kähler metrics on $X$ collapse to $Y$ in a weak sense. More recently, Gross et al. [28] proved that if the smooth fibers are tori then the collapsing happens in the $C^{\infty}$ topology, and in [29] we identified the Gromov-Hausdorff limit when $Y$ is a curve.

The question which we study in this paper, which arose from these works, is whether one can classify such fibrations when no singular fibers are allowed. For such manifolds the smooth collapsing was proved by Fine [16]. Here we show that these fibrations are very special. Our first result is: 
Theorem 1.1. If $f: X \rightarrow Y$ is a holomorphic submersion with connected fibers between projective manifolds with $K_{X} \cong \mathcal{O}_{X}$, then there is a finite unramified covering $\tilde{Y} \rightarrow Y$ with $K_{\tilde{Y}} \cong \mathcal{O}_{\tilde{Y}}$ such that the pullback family $X \times_{Y} \tilde{Y} \rightarrow \tilde{Y}$ is trivial, i.e., it is biholomorphic to the product family $\tilde{Y} \times$ $F \rightarrow \tilde{Y}$, where $F$ is a projective manifold with trivial canonical bundle.

In particular, this implies that the canonical bundle of $Y$ is holomorphically torsion, which is not a priori clear. As a simple consequence we have:

Theorem 1.2. If $f: X \rightarrow Y$ is a holomorphic submersion with connected fibers between projective manifolds with $X$ Calabi-Yau, then there are projective manifolds $B, F$ with trivial canonical bundles, and finite unramified coverings $g: B \times F \rightarrow X$ and $h: B \rightarrow Y$ such that the diagram

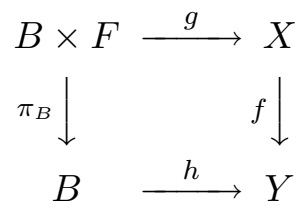

commutes.

If we drop the projectivity assumption, then the statement of Theorem 1.1 is false (see Remark 4.2 below), and has to be modified as follows:

Theorem 1.3. If $f: X \rightarrow Y$ is a holomorphic submersion with connected fibers between compact Kähler manifolds with $K_{X} \cong \mathcal{O}_{X}$, then $f$ is a holomorphic fiber bundle with fiber $F$ a compact Kähler manifold with $K_{F} \cong \mathcal{O}_{F}$ and with base $Y$ Calabi-Yau. If furthermore either $b_{1}(F)=0$ or $F$ is a torus and $b_{1}(Y)=0$, then there is a finite unramified covering $\tilde{Y} \rightarrow Y$ with $K_{\tilde{Y}} \cong \mathcal{O}_{\tilde{Y}}$ such that the pullback bundle $X \times_{Y} \tilde{Y} \rightarrow \tilde{Y}$ is holomorphically trivial.

In the literature, there are several related results. For example Fang and $\mathrm{Lu}$ [15, Corollary 1.3] have shown that if the fibers of $f$ are primitive Calabi-Yau manifolds which satisfy a certain cohomological assumption, then the family is isotrivial (in the sense that all fibers over a Zariski open set of $Y$ are biholomorphic to each other). This result holds without the assumption that $X$ is Calabi-Yau. More recently, Zhang-Zuo [43, Corollary 2.5] showed that if $Y$ is simply connected and $X$ has trivial canonical bundle and $h^{p}\left(X, \mathcal{O}_{X}\right)=0$ for $0<p<n$, then the family is isotrivial. In the setup 
of Theorem 1.1, if one knows in addition that $\kappa(Y) \geqslant 0$, then an alternative proof of the theorem can be given using the canonical bundle formula [22] and arguing along the lines of [1, Theorem 4.8] (see also Remark 3.4 below). If one knows furthermore that $Y$ is Calabi-Yau, then the conclusion that $f$ is a bundle also follows from [4, Theorem 2] (see also [11, Theorem 3.1]).

On the other hand, we make no assumptions on $Y$, and we first show that $Y$ has a Kähler metric with nonnegative Ricci curvature. Using Hodge theory, we get a holomorphic period map from the universal cover of $Y$ to a classifying space $\mathcal{D}$ of polarized real Hodge structures. This space has a Hermitian metric with strictly negative holomorphic sectional curvature in the horizontal directions. Using a version of Yau's Schwarz Lemma [41] we show that the period map is constant, and the infinitesimal Torelli theorem for Calabi-Yau manifolds implies that all fibers are biholomorphic, and then the main theorem follows.

This paper is organized as follows: in Section 2 we deduce Theorem 1.2 from Theorem 1.1, and show that to prove Theorem 1.1 it is enough to prove that all fibers are biholomorphic. This statement is then proved in Section 3. Theorem 1.3 is proved in Section 4.

\section{Preliminary results}

Proof of Theorem 1.2 assuming Theorem 1.1. Since $K_{X}$ is holomorphically torsion, there is a finite unramified covering $X^{\prime} \rightarrow X$ with $K_{X^{\prime}}$ holomorphically trivial. The composition $f^{\prime}: X^{\prime} \rightarrow X \rightarrow Y$ is a holomorphic submersion, but its fibers might be disconnected. Consider its Stein factorization $X^{\prime} \stackrel{f^{\prime \prime}}{\rightarrow} Y^{\prime} \stackrel{p}{\rightarrow} Y$, which has the properties that $Y^{\prime}$ is a projective manifold, $p$ is a finite unramified covering and $f^{\prime \prime}$ is a holomorphic submersion with connected fibers (see e.g., [21, Lemma 2.4]). Therefore $f^{\prime \prime}: X^{\prime} \rightarrow Y^{\prime}$ satisfies the hypotheses of Theorem 1.1, and hence there is a finite unramified covering $\tilde{Y} \rightarrow Y^{\prime}$ such that the pullback family $X^{\prime} \times_{Y^{\prime}} \tilde{Y} \rightarrow \tilde{Y}$ is trivial. We let $B=\tilde{Y}$ and let $h: B \rightarrow Y$ be the composition $\tilde{Y} \rightarrow Y^{\prime} \rightarrow Y$. We have a biholomorphism $X^{\prime} \times_{Y^{\prime}} \tilde{Y} \cong B \times F$, which composed with the map $X^{\prime} \times_{Y^{\prime}} \tilde{Y} \rightarrow X^{\prime} \rightarrow X$ gives us a finite unramified covering map $g: B \times F \rightarrow$ $X$, and Theorem 1.2 follows.

Note that from Theorem 1.1 it follows that the fibers $X_{y}$ of the original family must all be biholomorphic to each other. In fact, it is enough to prove this assertion to prove Theorem 1.1: 
Lemma 2.1. If the fibers $X_{y}$ of a family $f: X \rightarrow Y$ as in Theorem 1.1 are all biholomorphic to each other, then the conclusion of Theorem 1.1 holds.

Proof. Indeed, in this case we can apply the Fischer-Grauert theorem [17] and conclude that $f$ is a holomorphic fiber bundle, and then we can apply [32, Lemma 17] to get a finite unramified covering $\tilde{Y} \rightarrow Y$ such that the pullback family $X \times_{Y} \tilde{Y} \rightarrow \tilde{Y}$ is trivial. Now $X \times_{Y} \tilde{Y} \rightarrow X$ is also a finite unramified covering, hence the canonical bundle of $X \times_{Y} \tilde{Y}$ is holomorphically trivial. But $X \times_{Y} \tilde{Y} \cong \tilde{Y} \times F$, and so the canonical bundles of $\tilde{Y}$ and $F$ are both trivial.

In this proof we used [32, Lemma 17] which uses crucially the assumption that $X$ is projective. In fact, [32, Lemma 17] is false if $X$ is only compact Kähler, see Remark 4.2. However, as we will see in Section 4, [32, Lemma 17] is true in the Kähler case if either $b_{1}(F)=0$ or $F$ is a torus and $b_{1}(Y)=0$.

Thus, to prove Theorem 1.1, we are reduced to showing that all the fibers are biholomorphic. In the case when $\operatorname{dim} Y=\operatorname{dim} X-1$ the proof is very simple: in this case the fibers $X_{y}$ are elliptic curves, which are classified by their $j$-invariant, and mapping $y$ to the $j$-invariant of $X_{y}$ gives a holomorphic function on $Y$ which must be constant since $Y$ is compact. We follow a similar approach in higher dimensions, which is necessarily more complicated.

\section{Proof of theorem 1.1}

The main result of this section is the following:

Theorem 3.1. If $f: X \rightarrow Y$ is a holomorphic submersion between compact Kähler manifolds with $K_{X} \cong \mathcal{O}_{X}$, then $f$ is a holomorphic fiber bundle with fiber $F$ a compact Kähler manifold with $K_{F} \cong \mathcal{O}_{F}$ and with base $Y$ CalabiYau.

In particular, since all fibers of a holomorphic fiber bundles are biholomorphic, Theorem 1.1 follows immediately from Theorem 3.1 and Lemma 2.1 .

Proof. Let $\omega_{X}$ be a Kähler metric on $X$, and note that since the canonical bundle $K_{X}$ is trivial, the fibers $X_{y}=f^{-1}(y)$ also have trivial canonical bundle. Thanks to Tosatti [39, Proposition 4.1] (see also [36]), there is a Kähler metric $\omega$ on $Y$ such that $\operatorname{Ric}(\omega)=\omega_{W P} \geqslant 0$, where $\omega_{W P}$ is a Weil-Petersson type semipositive definite form [20]. In fact, more generally, it is true that for 
$Y$ to admit a Kähler metric with nonnegative Ricci curvature, it is enough to assume that $X$ admits such a metric, thanks to a result of Berndtsson ([3, Theorem 1.2] applied with $L=-K_{X}$ ).

We now need to use real polarized Hodge structures. These are defined without any projectivity assumption, by a straightforward generalization of Griffiths' original definition in the projective case [24]. Here we follow the discussion in [23, Section 8.1] (see also [13, Definition 1.5]). Let $P \subset$ $H^{n-m}\left(X_{y}, \mathbb{C}\right)=: H$ be the primitive cohomology induced by the Kähler class $\left[\left.\omega_{X}\right|_{X_{y}}\right]$. Let $H_{\mathbb{R}}=P \cap H^{n-m}\left(X_{y}, \mathbb{R}\right), h^{p, q}=\operatorname{dim}_{\mathbb{C}} P \cap H^{q}\left(X_{y}, \Omega_{X_{y}}^{p}\right)$ for $p+$ $q=n-m$, and call $Q$ the quadratic form on $H$ given by

$$
Q(\phi, \psi)=(-1)^{\frac{(n-m)(n-m-1)}{2}} \int_{X_{y}} \phi \wedge \psi
$$

which is called a polarization (even though no integrality assumption is made).

Then the construction by Griffiths [25, Section 8] gives a classifying space $\mathcal{D}$ for real polarized Hodge structure of type $\left\{H_{\mathbb{R}}, h^{p, q}, Q\right\}$, and a well-defined holomorphic period map $\mathcal{P}: \tilde{Y} \rightarrow \mathcal{D}$, where $\tilde{Y}$ is the universal cover of $Y$. If $\pi: \tilde{Y} \rightarrow Y$ is the universal covering map, and $y=\pi(z)$, then we have $\mathcal{P}(z)=\left\{P^{p, q}=P \cap H^{q}\left(X_{y}, \Omega_{X_{y}}^{p}\right)\right\}$. Furthermore, $\mathcal{P}$ is horizontal in the sense that $\mathcal{P}_{*}(T(\tilde{Y})) \subset T_{h}(\mathcal{D})$, where $T_{h}(\mathcal{D})$ is the horizontal subbundle of $T(\mathcal{D}) \subset \oplus_{r>0} \operatorname{Hom}\left(P^{p, q}, P^{p-r, q+r}\right)$ defined, for example, in Definition 25 of Chapter I in [26].

There is a Hermitian metric $\omega_{H}$ on $\mathcal{D}$ such that for any unit vector $\xi \in T_{h}(\mathcal{D})$, its holomorphic sectional curvature satisfies

$$
R(\xi, \bar{\xi}, \xi, \bar{\xi}) \leqslant-A<0
$$

for a constant $A>0$ ([27, Theorem 9.1], [13, Theorem 5.16] or Chapter II in [26]). In fact, the restriction of the metric $\omega_{H}$ to any horizontal slice is Kähler thanks to $\mathrm{Lu}$ [34], but we would not need this fact.

If we let $\tilde{\omega}=\pi^{*} \omega$, then $\tilde{\omega}$ is a complete Kähler metric on $\tilde{Y}$ with nonnegative Ricci curvature. We then apply the Schwarz Lemma 3.3 below to conclude that $\mathcal{P}: \tilde{Y} \rightarrow \mathcal{D}$ is constant.

By [24, Theorem 1.27], [10, Theorem 5.3.4] or Chapter III in [26], $\mathcal{P}_{*, z}$ : $T_{z} \tilde{Y} \rightarrow T_{h}(\mathcal{D})_{\mathcal{P}(z)}$ is a composition of the Kodaira-Spencer map $\rho: T_{z} \tilde{Y} \rightarrow$ $H^{1}\left(X_{y}, \Theta_{X_{y}}\right),(y=\pi(z))$, and the cup product map $w: H^{1}\left(X_{y}, \Theta_{X_{y}}\right) \rightarrow$ $T_{h}(\mathcal{D})_{\mathcal{P}(z)}$, where $\Theta_{X_{y}}$ is the sheaf of holomorphic vector fields on $X_{y}$. Since $X_{y}$ has trivial canonical bundle, $w$ is injective (Example 5.6.2 in [10] or [24, Proposition 3.6], [7], [37]). This result is also known as the infinitesimal 
Torelli theorem for Calabi-Yau manifolds. Thus the Kodaira-Spencer map $\rho$ of the pullback family over $\tilde{Y}$ is trivial. This implies that the KodairaSpencer map of the family over $Y$ is trivial too. Now the fibers $X_{y}$ have trivial canonical bundle, and hence $H^{1}\left(X_{y}, \Theta_{X_{y}}\right) \cong H^{1, n-1}\left(X_{y}\right)$ has dimension independent of $y \in Y$. Therefore thanks to Kodaira [31, Theorem 4.6] the complex structure of $X_{y}$ does not change when $y$ varies, i.e., all fibers $X_{y}$ are biholomorphic to each other. The Fischer-Grauert theorem [17] implies that $f$ is a holomorphic fiber bundle with fiber $F \cong X_{y}$ with trivial canonical bundle. Since all fibers $X_{y}$ are biholomorphic to each other, the WeilPetersson form $\omega_{W P}$ of the fibration $f$ vanishes identically. Its cohomology class is $\left[\omega_{W P}\right]=c_{1}(Y)$, hence $Y$ is Calabi-Yau.

Remark 3.2. The proof we just finished in fact shows the following result: if $f: X \rightarrow Y$ is a holomorphic submersion between compact Kähler manifolds with fibers $X_{y}$ with trivial canonical bundle and base $Y$ which admits a Kähler metric with nonnegative Ricci curvature then $f$ is a holomorphic fiber bundle.

In the proof of Theorem 3.1, we used the following improved version of Yau's Schwarz Lemma [41], which incorporates an observation of Royden [35] (see also [12, 38]).

Lemma 3.3 (Schwarz Lemma). Let $\left(X, \omega_{X}\right)$ be a complete Kähler manifold with nonnegative Ricci curvature and $\left(Y, \omega_{Y}\right)$ be a Hermitian manifold. Let $f: X \rightarrow Y$ be a holomorphic map, and assume that the holomorphic sectional curvature of $\omega_{Y}$ satisfies

$$
R_{Y}(\xi, \bar{\xi}, \xi, \bar{\xi}) \leqslant-A<0,
$$

for a constant $A>0$ and for all unit vectors $\xi$ in the image of $f_{*}: T^{1,0} X \rightarrow$ $T^{1,0} Y$. Then $f$ is constant.

Proof. Let $u=\operatorname{tr}_{g_{X}}\left(f^{*} g_{Y}\right)$, which is a smooth nonnegative function on $X$, and vanishes identically if and only if $f$ is constant. Assume that $f$ is not

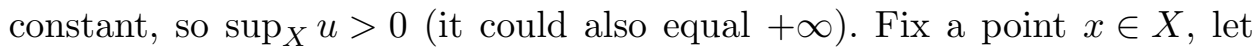
$y=f(x)$, and choose a local unitary coframe $\left\{\theta^{1}, \ldots, \theta^{n}\right\}$ for $g_{X}$ near $x$ and a local unitary coframe $\left\{\tilde{\theta}^{1}, \ldots, \tilde{\theta}^{m}\right\}$ for $g_{Y}$ near $y$. For any $0 \leqslant \alpha \leqslant m$ write

$$
f^{*} \tilde{\theta}^{\alpha}=\sum_{i} f_{i}^{\alpha} \theta^{i}
$$


so $\left\{f_{i}^{\alpha}\right\}$ are the components of $f_{*}$ in the chosen coframes. Therefore we have $u(x)=\sum_{\alpha, i}\left|f_{i}^{\alpha}\right|^{2}$. Let $\Delta_{g_{X}}=g^{i \bar{j}} \partial_{i} \partial_{\bar{j}}$ be the (complex) Laplacian of $g_{X}$ acting on functions. Then a direct calculation (see, e.g., [38, (5.9)], noting that the Laplacian used there is twice the complex Laplacian) shows that at $x$ we have

$$
\begin{aligned}
\Delta_{g_{X}} u & \geqslant \sum_{\alpha, i, j} \operatorname{Ric}\left(g_{X}\right)_{i \bar{j}} \overline{f_{i}^{\alpha}} f_{j}^{\alpha}-\sum_{\alpha, \beta, \gamma, \delta, i, k}\left(R_{Y}\right)_{\alpha \bar{\beta} \gamma \bar{\delta}} f_{i}^{\alpha} \overline{f_{i}^{\beta}} f_{k}^{\gamma} \overline{f_{k}^{\delta}} \\
& \geqslant-\sum_{i, k} R_{Y}\left(\xi_{i}, \overline{\xi_{i}}, \xi_{k}, \overline{\xi_{k}}\right)
\end{aligned}
$$

using the assumption that $\operatorname{Ric}\left(g_{X}\right) \geqslant 0$, where $\xi_{i}=f_{*}\left(e_{i}\right)$ and $\left\{e_{1}, \ldots, e_{n}\right\}$ is the unitary frame for $g$ dual to the coframe $\left\{\theta^{i}\right\}$. By changing the unitary frames at $x$ and $y$ if necessary, we may assume that the vectors $\left\{\xi_{i}\right\}$ are pairwise orthogonal. Assume that $f$ is not constant at $x$, so that the rank of $\left(f_{*}\right)_{x}$ is strictly positive. Then the assumption (3.3) together with an inequality of Royden [35, Lemma, p. 552] gives that

$$
\sum_{i, k} R_{Y}\left(\xi_{i}, \overline{\xi_{i}}, \xi_{k}, \overline{\xi_{k}}\right) \leqslant-A\left(\sum_{i}\left\|\xi_{i}\right\|_{g_{Y}}^{2}\right)^{2}=-A\left(\sum_{\alpha, i}\left|f_{i}^{\alpha}\right|^{2}\right)^{2}=-A u^{2}
$$

Hence at $x$ we have

$$
\Delta_{g_{X}} u \geqslant A u^{2}
$$

We now conclude the proof exactly as in [38, 41]. The generalized maximum principle of Yau (see, e.g., [38, Proposition 4.1]) shows that if $v$ is a smooth function on $X$ which is bounded below, then given any $\varepsilon>0$ there is a point $x_{\varepsilon} \in X$ with $\Delta_{g_{X}} v\left(x_{\varepsilon}\right) \geqslant-\varepsilon,\|d u\|_{g_{X}}\left(x_{\varepsilon}\right) \leqslant \varepsilon$ and $\liminf _{\varepsilon \rightarrow 0} v\left(x_{\varepsilon}\right)=\inf _{X} v$. We apply this to $v=(u+1)^{-1 / 2}$, so that $\limsup _{\varepsilon \rightarrow 0} u\left(x_{\varepsilon}\right)=\sup _{X} u>0$, and we get

$$
\begin{aligned}
-\varepsilon \leqslant & \Delta_{g_{X}} v\left(x_{\varepsilon}\right)=-\frac{1}{2}\left(u\left(x_{\varepsilon}\right)+1\right)^{-3 / 2} \Delta_{g_{X}} u\left(x_{\varepsilon}\right) \\
& +\frac{3}{4}\left(u\left(x_{\varepsilon}\right)+1\right)^{-5 / 2}\|d u\|_{g_{X}}^{2}\left(x_{\varepsilon}\right) \\
\leqslant & -\frac{A}{2}\left(u\left(x_{\varepsilon}\right)+1\right)^{-3 / 2} u\left(x_{\varepsilon}\right)^{2}+\frac{3 \varepsilon^{2}}{4},
\end{aligned}
$$

which holds for all $\varepsilon>0$ such that $u\left(x_{\varepsilon}\right)>0$, so that $(3.4)$ holds at $x_{\varepsilon}$. Taking $\varepsilon$ arbitrarily close to 0 , we conclude that $\sup _{X} u=0$, which is a contradiction. 
Remark 3.4. Andreas Höring has communicated to us an alternative line of proof of Theorem 3.1 in the projective case, which uses rather different ingredients. Here is a brief sketch of the argument. If the base $Y$ is not uniruled, then $K_{Y}$ is pseudoeffective thanks to [5, Corollary 0.3], and one can conclude using the canonical bundle formula [22] and arguing along the lines of $[1$, Theorem 4.8].

If $Y$ is uniruled, take $\mathbb{P}^{1} \subset Y$ a free rational curve (i.e., with globally generated normal bundle). The pullback family $X^{\prime}=\left(X \times_{Y} \mathbb{P}^{1}\right) \rightarrow \mathbb{P}^{1}$ is such that $X^{\prime}$ is smooth, with nonnegative Kodaira dimension (by adjunction), and $X^{\prime} \rightarrow \mathbb{P}^{1}$ is a submersion. This contradicts [40, Theorem 0.2].

\section{The Kähler case}

In this section, we will prove the following result, which is a substitute for Lemma 2.1 with no projectivity assumption.

Theorem 4.1. Let $X, Y$ be compact Kähler manifolds and $f: X \rightarrow Y a$ holomorphic fiber bundle with base $Y$ and fiber $F$ Calabi-Yau manifolds. If either $b_{1}(F)=0$ or $F$ is a torus and $b_{1}(Y)=0$, then there is a finite unramified covering $\tilde{Y} \rightarrow Y$ such that the pullback bundle to $\tilde{Y}$ is holomorphically trivial.

Theorem 1.3 follows immediately from this result together with Theorem 3.1. In fact, in Theorem 4.1 we do not even assume that $X$ is Calabi-Yau.

Remark 4.2. Theorem 4.1, and hence Lemma 2.1 and Theorems 1.2 and 1.1, are false if $X$ is not projective and $b_{1}(F)>0$ and $b_{1}(Y)>0$. Indeed, let $\Lambda$ be the lattice in $\mathbb{C}^{2}$ spanned by the vectors $(1,0),(0,1),(i, 0),(i \sqrt{2}, i)$, and let $X=\mathbb{C}^{2} / \Lambda$. Then the torus $X$ has algebraic dimension 1 (see, e.g., [14, p. 50]), and its algebraic reduction map is $f: X \rightarrow Y$ with $Y$ an elliptic curve. Then $f$ is an elliptic bundle, which is not trivial (otherwise $X \cong Y \times F$ would have algebraic dimension 2). Since the algebraic dimension is invariant under finite unramified coverings, the bundle does not become trivial even after pulling back via a finite unramified covering $\tilde{Y} \rightarrow Y$.

Proof of Theorem 4.1. Fix $\omega$ a Kähler metric on $X$, let $\operatorname{Aut}^{0}(F)$ be the connected component of the identity of the automorphism group of $F$, and let $\operatorname{Aut}\left(F,\left[\left.\omega\right|_{F}\right]\right)$ be the group of automorphisms of $F$ which preserve the Kähler class $\left[\left.\omega\right|_{F}\right]$. The structure group of the bundle $f: X \rightarrow Y$ reduces to $\operatorname{Aut}\left(F,\left[\left.\omega\right|_{F}\right]\right)$. Thanks to Lieberman [33, Proposition 2.2] or [18, Theorem 
4.8], the quotient $\operatorname{Aut}\left(F,\left[\left.\omega\right|_{F}\right]\right) / \operatorname{Aut}^{0}(F)$ is finite. Taking a finite unramified covering of $Y$ we can then assume that the structure group reduces to $\operatorname{Aut}^{0}(F)$.

If we are in the case when $b_{1}(F)=0$, then we have that $\operatorname{Aut}^{0}(F)=$ $\{1\}$ and so the structure group of the bundle is trivial, and the bundle is holomorphically trivial.

We now treat the case when $F$ is a torus and $b_{1}(Y)=0$. Since $Y$ is Calabi-Yau, the Beauville-Bogomolov-Calabi decomposition theorem $[2,4$, 8] shows that $\pi_{1}(Y)$ is finite, so up to a finite unramified covering of $Y$ we can assume that $Y$ is simply connected. Since $F$ is a torus, we have that $\operatorname{Aut}^{0}(F) \cong F$ and so $f: X \rightarrow Y$ is a principal torus bundle. If we call $\mathcal{F}$ the sheaf over $Y$ of germs of local holomorphic maps from $Y$ to $F$, then principal bundles over $Y$ with fiber $F$ are classified by $H^{1}(Y, \mathcal{F})$, and we will write $\zeta \in H^{1}(Y, \mathcal{F})$ for the class of our bundle. If we write $F=\mathbb{C}^{n} / \Lambda$ with $\Lambda \cong \mathbb{Z}^{2 n}$, then the short exact sequence

$$
0 \rightarrow \Lambda \rightarrow \mathbb{C}^{n} \rightarrow F \rightarrow 0
$$

gives a long exact sequence

$$
H^{1}(Y, \Lambda) \rightarrow H^{1}(Y, \mathcal{O})^{n} \rightarrow H^{1}(Y, \mathcal{F}) \stackrel{c}{\rightarrow} H^{2}(Y, \Lambda) \rightarrow H^{2}(Y, \mathcal{O})^{n}
$$

where we call $c(\zeta)$ the Chern class of the bundle. $Y$ is simply connected implies $H^{1}(Y, \mathcal{O})^{n}=0$. On the other hand, the fact that $X$ is Kähler implies that $c(\zeta)$ is a torsion class, thanks to a theorem of Blanchard [6] (see also [30, Theorem 1.7]). But $H^{2}(Y, \Lambda)$ is torsion-free since $H_{1}(Y, \mathbb{Z})=0$, and hence $c(\zeta)=0$, and the principal bundle is holomorphically trivial.

Remark 4.3. If we assume that $f: X \rightarrow Y$ is a holomorphic fiber bundle with base $Y$ and fiber $F$ Calabi-Yau manifolds, with $b_{1}(Y)=0$, but $F$ not necessarily a torus, then we can conclude that $f$ is equal to the composition of a holomorphic fiber bundle with Calabi-Yau fiber over $Y \times T$ where $T$ is a torus, composed with the trivial bundle $Y \times T \rightarrow Y$.

Indeed, Campana [9] and Fujiki [19] constructed a relative Albanese map for $f: X \rightarrow Y$ which is a commutative diagram of compact complex manifolds and holomorphic maps

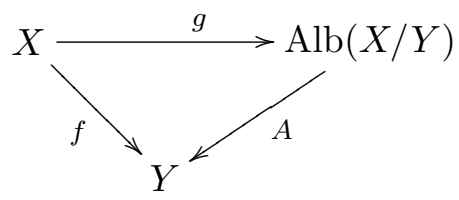


where $A: \operatorname{Alb}(X / Y) \rightarrow Y$ is a smooth submersion with fiber $A^{-1}(y) \cong$ $\operatorname{Alb}\left(X_{y}\right)$ the Albanese torus of $X_{y}=f^{-1}(y) \cong F$, and with $\left.g\right|_{X_{y}}: X_{y} \rightarrow$ $A^{-1}(y)$ isomorphic to the Albanese map $A_{y}: X_{y} \rightarrow \operatorname{Alb}\left(X_{y}\right)$ (for all $y \in Y$ ). Now all fibers $X_{y}$ are biholomorphic to $F$, and so their Albanese tori are all biholomorphic to $\operatorname{Alb}(F)$, and therefore $A$ is a holomorphic fiber bundle by the Fischer-Grauert theorem [17]. Since the fiber $X_{y} \cong F$ is Calabi-Yau, its Albanese map $A_{y}$ is a holomorphic fiber bundle with connected fiber $F_{1}$ which is Calabi-Yau, thanks to [8].

We claim now that $g$ is a submersion. Indeed, pick any point $z \in$ $\operatorname{Alb}(X / Y)$, let $y=A(z)$, and let $V$ be any tangent vector to $\operatorname{Alb}(X / Y)$ at $z$. Then $A_{*} V=0$ if and only if $V$ is tangent to $\operatorname{Alb}\left(X_{y}\right)$ (the fiber of $A$ ). Since $f$ is a submersion, we can find a vector $W$ tangent to $X$ at some point $x$ in the fiber $X_{y}$ such that $f_{*} W=A_{*} V$. Hence $A_{*}\left(g_{*} W-V\right)=0$, i.e., $g_{*} W-V$ is tangent to $\operatorname{Alb}\left(X_{y}\right)$. Since $\left.g\right|_{X_{y}}$ is isomorphic to $A_{y}: X_{y} \rightarrow \operatorname{Alb}\left(X_{y}\right)$ which is a bundle, there is a vector $Z$ tangent to $X$ at $x \in X_{y}$ with $g_{*} Z=g_{*} W-V$, and so $g_{*}(W-Z)=V$ and $g$ is a submersion. In particular, since $X$ is Kähler, we see that $\operatorname{Alb}(X / Y)$ is Kähler too.

Since all fibers of $g$ are biholomorphic to $F_{1}$, the Fischer-Grauert theorem implies that $g$ is a holomorphic fiber bundle. Now $A: \operatorname{Alb}(X / Y) \rightarrow Y$ is a holomorphic fiber bundle with total space Kähler, $Y$ simply connected, and fiber a torus, hence this bundle must be trivial thanks to Theorem 4.1. We thus obtain a new diagram

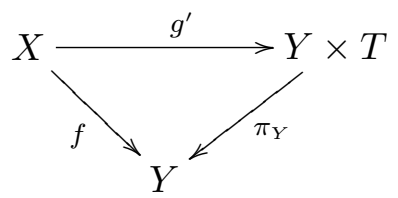

where $T$ is a torus and $g^{\prime}$ is still a holomorphic fiber bundle with fiber $F_{1}$ Calabi-Yau.

\section{Acknowledgments}

The first named author (Valentino Tosatti) is grateful to Florin Ambro, Keiji Oguiso and Xiaowei Wang for useful communications. The second named author (Yuguang Zhang) thanks Professors Mark Gross and Zhiqin Lu for some discussions. Both authors are grateful to Andreas Höring for communicating the content of Remark 3.4, and to the referee for useful suggestions. Valentino Tosatti Supported in part by a Sloan Research Fellowship and NSF 
grants DMS-1236969 and DMS-1308988. Yuguang Zhang was supported in part by NSFC-11271015.

\section{References}

[1] F. Ambro, The moduli b-divisor of an lc-trivial fibration, Compos. Math. 141(2) (2005), 385-403.

[2] A. Beauville, Variétés Kähleriennes dont la première classe de Chern est nulle, J. Differential Geom. 18(4) (1983), 755-782.

[3] B. Berndtsson, Curvature of vector bundles associated to holomorphic fibrations, Ann. Math. (2) $\mathbf{1 6 9}(2)$ (2009), 531-560.

[4] F.A. Bogomolov, Kähler manifolds with trivial canonical class, Math. USSR Izv. 8(1) (1974), 9-20.

[5] S. Boucksom, J.-P. Demailly, M. Păun and T. Peternell, The pseudoeffective cone of a compact Kähler manifold and varieties of negative Kodaira dimension, J. Algebraic Geom. 22 (2013), 201-248.

[6] A. Blanchard, Sur les variétés analytiques complexes, Ann. Sci. École Norm. Sup. (3) 73 (1956), 157-202.

[7] R.L. Bryant and P.A. Griffiths, Some observations on the infinitesimal period relations for regular threefolds with trivial canonical bundle, in Arithmetic and geometry, Vol. II, 77-102, Progr. Math., 36, Birkhäuser, Boston, 1983.

[8] E. Calabi, On Kähler manifolds with vanishing canonical class, in Algebraic geometry and topology. A symposium in honor of S. Lefschetz, 78-89. Princeton University Press, Princeton, N.J., 1957.

[9] F. Campana, Réduction d'Albanèse d'un morphisme propre et faiblement kählérien. I, Compositio Math. 54(3) (1985), 373-398.

[10] J. Carlson, S. Müller-Stach and C. Peters, Period mappings and period domains, Cambridge University Press, 2003.

[11] F. Catanese, K. Oguiso and T. Peternell, On volume-preserving complex structures on real tori, Kyoto J. Math. 50(4) (2010), 753-775.

[12] Z. Chen, S.-Y. Cheng and Q. Lu, On the Schwarz lemma for complete Kähler manifolds, Sci. Sin. 22(11) (1979), 1238-1247. 
[13] P. Deligne, Travaux de Griffiths, Sém. Bourbaki Exp. 376, 213-237, Lecture Notes in Mathematics, 180, Springer, Berlin, 1970.

[14] G. Elencwajg and O. Forster, Vector bundles on manifolds without divisors and a theorem on deformations, Ann. Inst. Fourier (Grenoble) 32(4) (1982), 25-51.

[15] H. Fang and Z. Lu, Generalized Hodge metrics and BCOV torsion on Calabi-Yau moduli, J. Reine Angew. Math. 588 (2005), 49-69.

[16] J. Fine, Fibrations with constant scalar curvature Kähler metrics and the CM-line bundle, Math. Res. Lett. 14(2) (2007), 239-247.

[17] W. Fischer and H. Grauert, Lokal-triviale Familien kompakter komplexer Mannigfaltigkeiten, Nachr. Akad. Wiss. Göttingen Math.-Phys. Kl. II (1965), 89-94.

[18] A. Fujiki, On automorphism groups of compact Kähler manifolds, Invent. Math. 44(3) (1978), 225-258.

[19] A. Fujiki, Relative algebraic reduction and relative Albanese map for a fiber space in $\mathcal{C}$, Publ. Res. Inst. Math. Sci. 19(1) (1983), 207-236.

[20] A. Fujiki and G. Schumacher, The moduli space of extremal compact Kähler manifolds and generalized Weil-Petersson metrics, Publ. Res. Inst. Math. Sci. 26(1) (1990), 101-183.

[21] O. Fujino and Y. Gongyo, On images of weak Fano manifolds, Math. Z. 270(1-2) (2012), 531-544.

[22] O. Fujino and S. Mori, A canonical bundle formula, J. Differential Geom. 56(1) (2000), 167-188.

[23] W.M. Goldman and J.J. Millson, The deformation theory of representations of fundamental groups of compact Kähler manifolds, Inst. Hautes Études Sci. Publ. Math. 67 (1988), 43-96.

[24] P.A. Griffiths, Periods of integrals on algebraic manifolds. II. Local study of the period mapping, Amer. J. Math. 90 (1968), 805-865.

[25] P.A. Griffiths, Periods of integrals on algebraic manifolds. III. Some global differential-geometric properties of the period mapping, Inst. Hautes Études Sci. Publ. Math. 38 (1970), 125-180.

[26] P.A. Griffiths, ed., Topics in transcendental algebraic geometry, Ann. Math. Stud., 106, Princeton University Press, Princeton, NJ, 1984. 
[27] P.A. Griffiths and W. Schmid, Locally homogeneous complex manifolds, Acta Math. 123 (1969), 253-302.

[28] M. Gross, V. Tosatti and Y. Zhang, Collapsing of abelian fibred CalabiYau manifolds, Duke Math. J. 162(3) (2013), 517-551.

[29] M. Gross, V. Tosatti and Y. Zhang, Gromov-Hausdorff collapsing of Calabi-Yau manifolds (2013), arXiv:1304.1820.

[30] T. Höfer, Remarks on torus principal bundles, J. Math. Kyoto Univ. 33(1) (1993), 227-259.

[31] K. Kodaira, Complex manifolds and deformation of complex structures, Springer-Verlag, Berlin, 2005.

[32] J. Kollár and M. Larsen, Quotients of Calabi-Yau varieties, in Algebra, arithmetic, and geometry in honor of Yu. I. Manin, Vol. II, 179-211, Progr. Math., 270, Birkhäuser Boston, Inc., Boston, MA, 2009.

[33] D.I. Lieberman, Compactness of the Chow scheme: applications to automorphisms and deformations of Kähler manifolds, 140-186, Lecture Notes in Mathematics, 670, Springer, Berlin, 1978.

[34] Z. Lu, On the geometry of classifying spaces and horizontal slices, Amer. J. Math. 121(1) (1999), 177-198.

[35] H.L. Royden, The Ahlfors-Schwarz lemma in several complex variables, Comment. Math. Helv. 55(4) (1980), 547-558.

[36] J. Song and G. Tian Canonical measures and Kähler-Ricci flow, J. Amer. Math. Soc. 25(2) (2012), 303-353.

[37] G.N. Tjurina, Deformation of complex structures on algebraic varieties, Soviet Math. Dokl. 4 (1963), 1567-1574.

[38] V. Tosatti, A general Schwarz Lemma for almost-Hermitian manifolds, Commun. Anal. Geom. 15(5) (2007), 1063-1086.

[39] V. Tosatti, Adiabatic limits of Ricci-flat Kähler metrics, J. Differential Geom. 84(2) (2010), 427-453.

[40] E. Viehweg and K. Zuo, On the isotriviality of families of projective manifolds over curves, J. Algebraic Geom. 10(4) (2001), 781-799.

[41] S.-T. Yau, A general Schwarz lemma for Kähler manifolds, Amer. J. Math. 100 (1978), 197-204. 
[42] S.-T. Yau, On the Ricci curvature of a compact Kähler manifold and the complex Monge-Ampère equation, I, Commun. Pure Appl. Math. 31(3) (1978), 339-411.

[43] Y. Zhang and K. Zuo, Calabi-Yau varieties with semi-stable fibre structures, Manuscr. Math. 126(3) (2008), 273-291.

Department of Mathematics

NORTHWESTERN UNIVERSITY

2033 SHERIDAN ROAD

EVANSTON, IL 60201

USA

E-mail address: tosatti@math.northwestern.edu

Mathematical Sciences Center

Tsinghua University

BEIJING 100084

People's Republic of China

E-mail address: yuguangzhang76@yahoo.com

Received October 1, 2013 